\title{
Dynamics of particles floating on liquid flowing in a semicircular open channel
}

\author{
S. Umeda * and W.-J. Yang \\ Department of Mechanical Engineering, and Applied Mechanics, The University of Michigan, Ann Arbor, MI 48109, USA
}

\begin{abstract}
The dynamic characteristics of surface-floating particles in liquids flowing in a two-dimensional, semicircular open channel is studied experimentally. For high visibility in the experiments, relatively large particles are employed whose particle-liquid density ratio is either equal to or less than unity. Particles of different size and geometry are tested in a water-glycerin mixture. A video camera traces the pathline of each particle from which the velocity and direction of particle motion are evaluated. Liquid velocity distribution is determined by hot-film anemometry. A modified dynamics (Basset-Boussinesq-Oseen) equation is derived and numerically solved by means of a finite-difference technique to determine fluid velocity. A new dimensionless parameter is disclosed which is pertinent to both particle geometry and fluid flow conditions. It correlates particle trajectory and velocity, trajectory dispersion and fluidparticle velocity ratio.
\end{abstract}

\section{Introduction}

The tracer method is one of many flow visualization techniques. For easier identification in image processing, one is tempted to select tracer particles of a size as large as possible within the range of tracking fluid motion (Merzkirch, 1987). In this case, although a larger particle diameter would make visualization easier, such a particle would encounter a problem of poor fluid-tracing characteristics. In addition to particle motion on the fluid surface, it is required that particles are neutrally buoyant in the fluid.

Aluminum flakes, lycopodium powder, wood sawdust, pulverized polyesters and other materials have been employed in the visualization of surface flow (Brandone and Bernard, 1971; Clayton and Massey, 1967; Dimotakis et al., 1981). Small aluminum flakes are susceptible to the influence of surface tension. Douglas et al. (1972) observed that the motion of a particle suspended in the fluid displays turbulent

* Visiting Scholar on leave from Department of Mechanical Engineering, Fukuyama University, Fukujama, Japan flow patterns beneath the liquid surface but that other particles floating on the free surface fail to indicate such flow variations. Gharib and Willert (1989) presented a comprehensive review on particle tracing and investigated the effect of curvature of particle trajectory on particle velocimetry.

In order to insure that the tracer is neutrally buoyant in a fluid, appropriate fluid-solid materials of equal density are selected. Tracer particles which match various liquids are compiled in a list (Merzkirch, 1987). In case of water, pliolite particles (made from polyvinyl toluene butadiene) of 10 to $200 \mu \mathrm{m}$ diameter are popularly employed. Tracer particles with diameter of about $100 \mu \mathrm{m}$ are commonly used in liquid mixtures. By introducing a special indicator in a fluid flow, the motion of the index represents the motion of a point in the fluid it occupies. However, the particle motion does not always coincide with the motion of fluid particle at all instants. Therefore, the understanding of tracer particles placed in a flow is very important in flow visualization.

The present study is based on the B. B. O. (Basset-Boussinesq-Oseen) equation of particle motion. A simple circular flow in a plane, two-dimensional bend is chosen as the liquid model to experimentally investigate the flow characteristics of different single particles with various different diameters which are lighter than a neutrally buoyant medium. Umeda and Yano (1988) investigated the characteristics of a suspended particle. They found that the submerged part plays an important role in the motion of a floating particle but have not arrived at complete understanding on the geometrical parameter of surface-floating particles in their theoretical analysis. Based on theoretical considerations, this study postulates a geometrical parameter which is most suitable to the B. B. O. equation for surface-floating particles. The validity of the geometrical parameter is confirmed through experiments. In addition, the relationship between the fluid flow and the particle pathline is disclosed. The velocity ratio of the tracer particle to the fluid is expressed in terms of the dimensionless parameter relating the particle geometry to the fluid velocity. In order to enhance the visualization accuracy in experiments, tracers are selected with the density ratio of less than unity and with relatively large diameters. 


\section{Theoretical analysis}

The motion of tracer particles suspended in a liquid is described by the force balance equation from which the particle velocity is determined. Consider a single spherical particle of radius $a$ with the velocity of $u_{p}$. If $U$, the difference between the fluid velocity of $u_{f}$ and $u_{p}$, is small, the B. B.O. equation reads (Soo, 1967).

$\frac{d u_{p}}{d t}=\frac{U}{\tau}-\frac{1}{\varrho_{p}} \frac{\partial p}{\partial r}+\frac{1}{2 \sigma} \frac{d U}{d t}+\frac{9}{2 \pi a \varrho_{p}}\left(\pi \varrho_{f} u\right)^{1 / 2} \int_{0}^{t} \frac{\frac{d U}{d t^{\prime}} d t^{\prime}}{\left(t-t^{\prime}\right)^{1 / 2}}+\frac{F_{e}}{\varrho_{p}}$

Here, $\varrho$ denotes the density; $t$, time; $p$, pressure; $r$, distance; $\mu$, absolute viscosity; $\sigma$, density ratio $\varrho_{p} / \varrho_{f} ; \tau$, time constant for momentum transfer due to drag force; and $F_{e}$, external force per unit volume of the particle in the potential field. The subscripts $p$ and $f$ correspond to particle and fluid, respectively. In a plane, two-dimensional field $F_{e}$ is considered negligible. $\tau$ can be expressed as by

$\tau=\frac{8 \sigma a}{3 C_{d}|U|}$

wherein $C_{d}$ represents the drag coefficient.

The B.B.O. equation is valid for small particles in a straight flow with a low relative velocity $U$ and neutrally buoyant. In contrast, the present study considers surfacefloating particles in a curved flow with a higher $U$. The objective of this study is to derive a modified B. B.O. equation to analyze the dynamic behavior of surface-floating particles of various geometry which are lighter than neutrally buoyant ones. The fraction of a particle immersed underneath the free surface varies with the density ratio of particle to liquid. Therefore, $C_{d}$ and $\tau$ for the surface-floating particles, even having an identical diameter, would have different values from those in Eqs. (1) and (2) for small submerged particles.

Since the action of buoyancy force is dependent upon the fluid-particle density ratio and particle mass, the draft $D_{r}$ is defined as the particle length that is immersed in the liquid as shown in Fig. 1. The maximum circumferential length, cross-sectional area and volume of a particle immersed in the liquid are $L_{p}, A_{p}$ and $V_{p}$, respectively. In addition to particles of spherical shape, the particles of cylindrical form are also tested. In the latter case, the maximum circumferential length $L_{p}$ is exactly the circumferential length irrespective of the draft.

Taking into account fluid resistance and

$-\frac{\partial p}{\partial r}=\varrho_{f} \frac{d u_{f}}{d t}$

the dynamics equation for surface-floating particles is derived as

$\frac{d u_{p}}{d t}=\frac{U}{\tau}+\frac{1}{\sigma} \frac{d u_{f}}{d t}+\frac{\alpha}{\sigma} \frac{d U}{d t}+\frac{6 A_{p}}{\pi \varrho_{p} V_{p}}\left(\pi \varrho_{f} \mu\right)^{1 / 2} \int_{0}^{t} \frac{d U / d t^{\prime}}{\left(t-t^{\prime}\right)^{1 / 2}} d t^{\prime}$

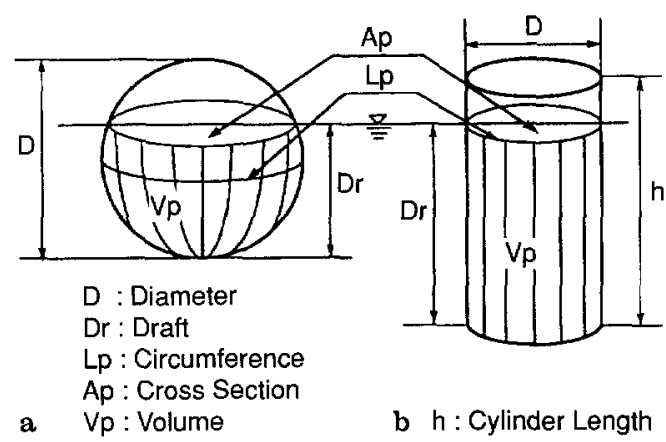

Fig. $1 \mathrm{a}$ and b. Definition of hydraulic quantities of a spherical and b cylindrical particles

The time constant in Eq. (2) becomes

$$
\left.\begin{array}{rlr}
\tau & =\frac{4 \sigma L_{p}}{3 \pi C_{d}|U|} \\
\alpha & =\frac{D_{r} A_{p}}{3 V_{p}} & \text { for sphere } \\
& =\frac{\mathrm{D}_{\mathrm{r}}}{\mathrm{h}}=\sigma & \text { for cylinder }
\end{array}\right\}
$$

in which $h$ signifies the cylinder length. Since the third term on the right side of Eq. (3) is the force to accelerate the apparent mass of the particle relative to the fluid, $\alpha$ may be considered the "shape factor" of the apparent mass of the surface-floating particle in liquid. Its physical interpretation is presented later in the "Results and discussion" section. In deriving Eq. (3), one should note that the effect of a free surface is small for the tested liquid (a water-glycerin mixture with the density of 1.16 and the kinematic viscosity of $0.16 \mathrm{~cm}^{2} / \mathrm{sec}$ ) flowing at a low velocity (less than $2 \mathrm{~cm} / \mathrm{s}$ ). In the case of partially-submerged cylindrical particles, they are carefully placed in the liquid to prevent the occurrence of tilting during flow.

Equations (3) and (4) are combined to yield

$$
\begin{aligned}
\frac{3 \pi C_{d}}{4 \sigma L_{p}} U|U|-\left(\frac{\sigma+\alpha}{\sigma}\right) \frac{d u_{p}}{d t} & +\left(\frac{1+\alpha}{\sigma}\right) \frac{d u_{f}}{d t} \\
& +\frac{6 A_{p}(\pi v)^{1 / 2}}{\pi \sigma V_{p}} \int_{0}^{t} \frac{\left(d U / d t^{\prime}\right) d t^{\prime}}{\left(t-t^{\prime}\right)^{1 / 2}}=0
\end{aligned}
$$

It is the modified B. B. O. equation for surface-floating particles. It should be emphasized that Eq. (6) is derived in accordance with the observed physical phenomena without referring to other sources (non-existing). The initial conditions are

$u_{p}(0)=u_{f}(0)=u_{f 0}$

in which $u_{f o}$ is the fluid velocity at the entrance to the curved channel. One defines the dimensionless time $t_{*}$ as

$t_{*}=t / T$ 
where $T$ is the characteristic time of the fluid-particle system defined as

$$
\begin{array}{rlr}
T & =\frac{2 A_{p}(\sigma+\alpha)}{9 \pi v} & \text { for spheres } \\
& =\frac{A_{p}(\sigma+\alpha)}{18 v} & \text { for cylinders }
\end{array}
$$

The drag coefficient is determined by means of Stoke's law as

$C_{d}=\frac{\gamma v L_{p}}{u_{p} A_{p}}$

Here, $\gamma$ takes the values of 6 and $24 / \pi$ for spheres and cylinders, respectively. For convenience, $u_{p}$ is used as the flow velocity in $C_{d}$. The substitution of Eq. (10) into Eq. (4) yields

$$
\begin{aligned}
\tau & =\frac{2 A_{p} \sigma u_{p}}{9 \pi v|U|} & \text { for spheres } \\
& =\frac{A_{p} \sigma u_{p}}{18 v|U|} & \text { for cylinders }
\end{aligned}
$$

For a special case in which a spherical particle suspends in the bulk of a flowing fluid, $D_{r}=2 a, L_{p}=2 \pi a, A_{p}=\pi a^{2}$, and $V_{p}=4 \pi a^{3} / 3$, therefore one finds

$$
\begin{aligned}
& \alpha=\frac{1}{2}, \quad T=\frac{2 \pi a^{2}(\sigma+1 / 2)}{9 \pi v}, \quad \operatorname{Re}=\frac{2 a u_{f}}{v}, \\
& C_{d}=\frac{12 v}{a u_{p}}, \quad \tau=\frac{2 \sigma a^{2} u_{p}}{9 v|U|}
\end{aligned}
$$

Equations (6) and (10) are reduced to Eqs. (1) and (2), respectively.

A combination of Eqs. (5), (8), (9) and (10) yields, for both spherical and cylindrical particles, a new parameter:

$\frac{C_{d}}{t_{*}}=\left(\frac{L_{p}}{u_{p} t}\right) \frac{4(\sigma+\alpha)}{3 \pi}=\gamma\left(\frac{D_{r} L_{p}}{A_{p}}\right) \frac{1}{\operatorname{Re} t_{*}}$

$u_{\mathrm{p}} t$ signifies the migration distance of a particle in time $t$. Hence, $L_{p} / u_{p} t$ is a dimensionless length signifying the ratio of the maximum circumferential length immersed in the liquid to the migration length of a particle. The other parameter $4(\sigma+\alpha) / 3 \pi$ includes the density ratio $\sigma$ and the "shape factor" of the apparent mass of a surface-floating particle $\alpha$. Therefore, $C_{d} / t_{*}$ must be an important dimensionless parameter for tracing the pathline of particles floating on the surface of a flowing fluid. The rationale will be experimentally examined in the present work. Equation (13) also suggests the involvement of $R e$ in $C_{d} / t_{*}$. The integro-differential Eq. (6) is operated on by means of a finite-difference method. It yields:

(i) for $U_{p, n+1} \leqq U_{p, n}$ :

$$
\begin{array}{r}
k_{1}\left(u_{f, n+1}-u_{p, n+1}\right)^{2}+\frac{k_{2}}{\Delta t}\left(u_{p, n+1}-u_{p, n}\right)+\frac{k_{3}}{\Delta t}\left(u_{f, n+1}-u_{f, n}\right) \\
+\frac{2 k_{4}}{(\Delta t)^{1 / 2}}\left[\left(u_{f, n+1}-u_{f, n}\right)-\left(u_{p, n+1}-u_{p, n}\right)\right]=0
\end{array}
$$

(ii) for $u_{p, n+1}>u_{p, n}$ :

$$
\begin{gathered}
k_{1}\left(u_{f, n+1}-u_{p, n+1}\right)^{2}-\frac{k_{2}}{\Delta t}\left(u_{p, n+1}-u_{p, n}\right)+\frac{k_{3}}{\Delta t}\left(u_{f, n+1}-u_{f, n}\right) \\
+\frac{2 k_{4}}{(\Delta t)^{1 / 2}}\left[\left(u_{f, n+1}-u_{f, n}\right)-\left(u_{p, n+1}-u_{p, n}\right)\right]=0
\end{gathered}
$$

Here, $\Delta t$ denotes the time step. The constants $k$ 's are defined as

$k_{1}=\frac{3 \pi C_{d}}{4 \sigma L_{p}}, k_{2}=\frac{\sigma+\alpha}{\sigma}, k_{3}=\frac{1+\alpha}{\sigma}, k_{4}=\frac{6 A_{p}(\pi v)^{1 / 2}}{\pi \sigma V_{p}}$

The subscripts $n$ and $n+1$ indicate the present and next time instants, respectively. The initial conditions are

$u_{f 1}=u_{p 1}$

(measured at entrance to the semicircular channel)

The particle velocity $u_{p}$ is obtained by an experimental measurement, while Eq. (14) or (15) determines the fluid velocity $u_{f}$.

\section{Experimental apparatus and procedure}

Figure 2 is a schematic of the experimental setup for studying the motion of tracer particles in the flowing fluid. It consisted of a semicircular channel with each end being connected to a straight channel. The bottom of the circular section was installed with a $0.5 \mathrm{~cm}$-interval mesh for convenience in tracing the pathline of each particle. Flow in the curved channel is subjected to centrifugal force and thus has a tendency to shift outward. Hence, a weir with gentle slope was installed at the end of the downstream straight channel to direct the flow-direction slightly inward.

Tests were conducted on twenty different combinations of different diameter, density ratio and draft for spherical particles. Nine combinations for cylindrical particles were employed in the tests. Table 1 shows the ranges of diameter, density ratio and draft.

The liquid used in the study was a water-glycerin mixture with the density ratio of 1.16 and the kinematic viscosity of $0.16 \mathrm{~cm}^{2} / \mathrm{sec}$. The liquid temperature was maintained at $25^{\circ} \mathrm{C}$. Six different flows, runs 1 to 6 , were tested with differ-

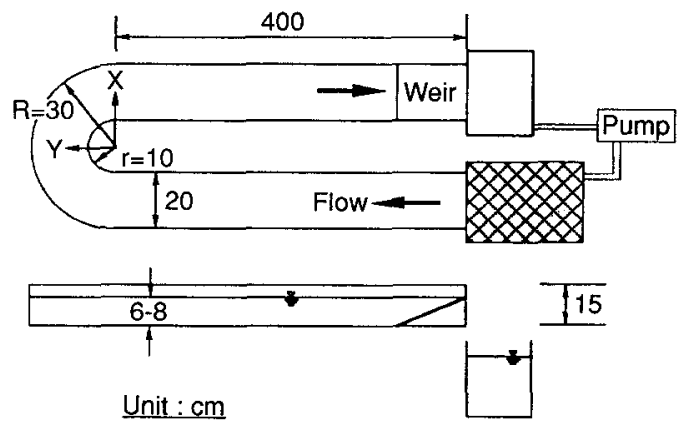

Fig. 2. A schematic of experimental setup 
Table 1. Conditions of spherical and cylindrical particles used in tests

\begin{tabular}{llll}
\hline Particle geometry & Diameter, cm & Density ratio & Draft, cm \\
\hline Sphere & 0.14 to 1.16 & 0.22 to 1.00 & 0.10 to 1.02 \\
Cyclinder & 0.29 to 0.91 & 0.78 to 0.96 & 1.60 to 2.01 \\
\hline
\end{tabular}

Table 2. A comparison of mean velocities of particle No. $2(D=$ $0.14 \mathrm{~cm}, D_{r}=0.10 \mathrm{~cm}$ and $\sigma=0.75$ ) in six test runs

\begin{tabular}{lllllll}
\hline Run number & 1 & 2 & 3 & 4 & 5 & 6 \\
\hline Mean velocity, cm/s & 0.80 & 0.88 & 1.40 & 1.47 & 1.48 & 1.68 \\
\hline
\end{tabular}

ent flow velocity and direction which were induced by varying the weir height. Table 2 compares the average velocities of the smallest spherical particle, No. 2, (particle diameter $D=0.14 \mathrm{~cm}, D_{r}=0.10 \mathrm{~cm}, \sigma=0.75$ ) near the entrance to the circular channel in the six test runs. A high liquid flow tends to induce a rigorous secondary stream in the channel, disrupting the stable movement of surface-floating particles.

A video camera was employed to photograph the pathline of each particle in the curved channel, from which the velocity and direction of particle motion at each point were determined. The liquid velocity distribution was measured using a hot-film type anemometer. The measurements were taken at eight locations; $2,4,6,8,10,13,15$ and $20 \mathrm{~mm}$ from the liquid surface. In the planar direction, measurements were performed at 250 points starting from the mesh points installed in the curved channel, densely measured in the vicinity of particle pathlines. The values of $\mu_{f}$ measured by a hot-film anemometer were almost uniform with depth near the free surface. The particle velocity $\mu_{p}$ was determined by measuring the movement of particles on the video recording over a given time duration.

Each particle was placed on the liquid surface in the channel center at several centimeters upstream from the entrance of the curved section. The placements of each particle were repeated about ten times. Only those which passed through the center of the entrance were selected for tracing their pathlines. Since the test liquid contained highly viscous glycerin, the effect of liquid diffusion was small, making the determination of an average pathline a relatively easy task.

\section{Results and discussion}

For convenience, the upstream half of the semicircular channel is divided into three zones of equal size ( 30 degrees each): Zones I, II and III, as illustrated in Fig. 3.

Since particles with the density ratio of less than unity are used in the study, the hydraulic quantities, $L_{p}, A_{p}, V_{p}$ and $D_{r}$, as defined in Fig. 1, are used. In view of the relationship between the fluid flow and the particle pathline, the drift of

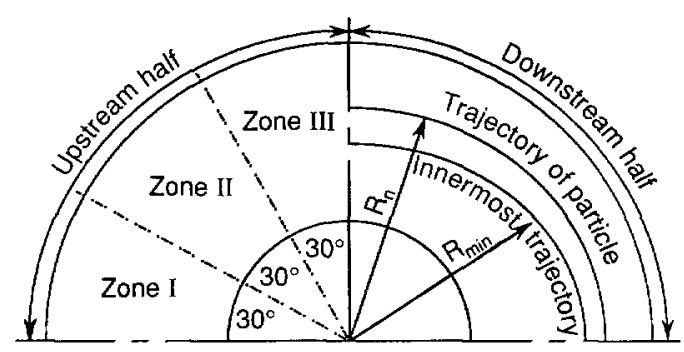

Fig. 3. Division of flow passage and dispersion of particle trajectories in the semicircular channel

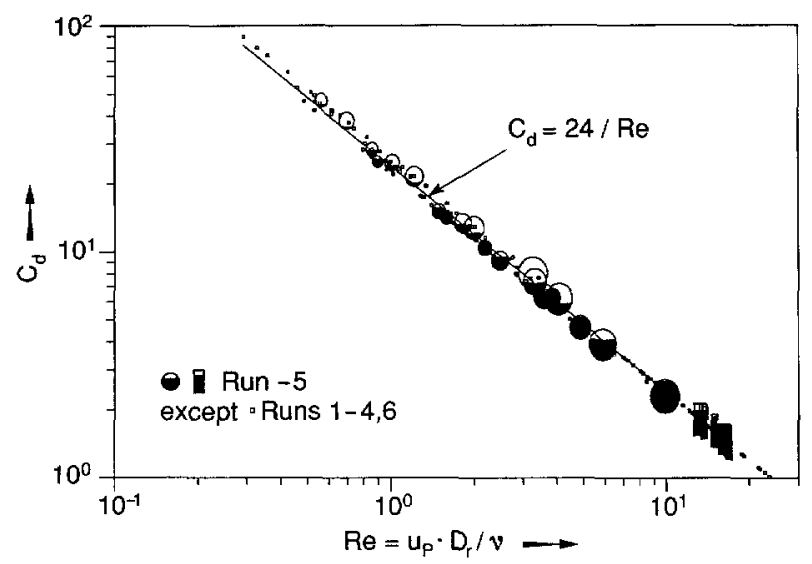

Fig. 4. A plot of drag coefficient $C_{d}$ versus particle Reynolds number $R e$ in zone I

surface-floating particles must have an extremely important bearing on flow fluid. Hence, the Reynolds number for describing such a two-phase flow is defined as $R e=u_{p} D_{r} / v$ using $D_{r}$ as the characteristic length, with $u_{p} \cong u_{f}$. Figure 4 illustrates its relationship with the drag coefficient $C_{d}$ of Eq. (10) in zone I. The circular and rectangular legends represent spherical and cylindrical particles, respectively. The immersed portions of their diameter and column length are shown in black (solid) and the exposed portion in white (hollow), in proportion to each specific value of the particlefluid density ratio. Different symbols are used for other runs to avoid possible confusion. The straight line is the Stoke's formula, $C_{d}=24 / R e$. All test data fall right on the line, indicating the validity of definition of the cross-sectional quantities. Same results are obtained for zones II and III (not shown here).

The "shape factor" $\alpha$ in Eq. (5) is defined as a measure of the submerged portion of a surface-floating particle. It varies with the density ratio of particle to fluid, $\sigma$. For $\sigma=1$, the particle is completely submerged and $\alpha=1$. A particle is less submerged as $\sigma$ is decreased from unity. The value of $\alpha$ is also dependent upon the dimension of a surface-floating particle in the vertical and horizontal directions. For example, $\alpha$ of a slender cylinder varies directly with the vertical dimension, i.e. the draft $D_{r}$. In the case of a sphere, $\alpha$ depends on both the vertical dimension, i.e. $D_{r}$, and the horizontal dimension, i.e. the average width $V_{p} / A_{p}$ of the submerged portion. 


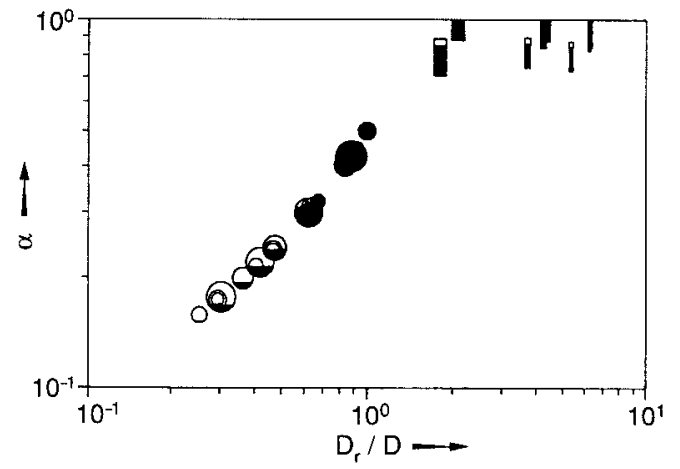

Fig. 5. A plot of "shape factor" $\alpha$ versus dimensionless draft $D_{r} / D$

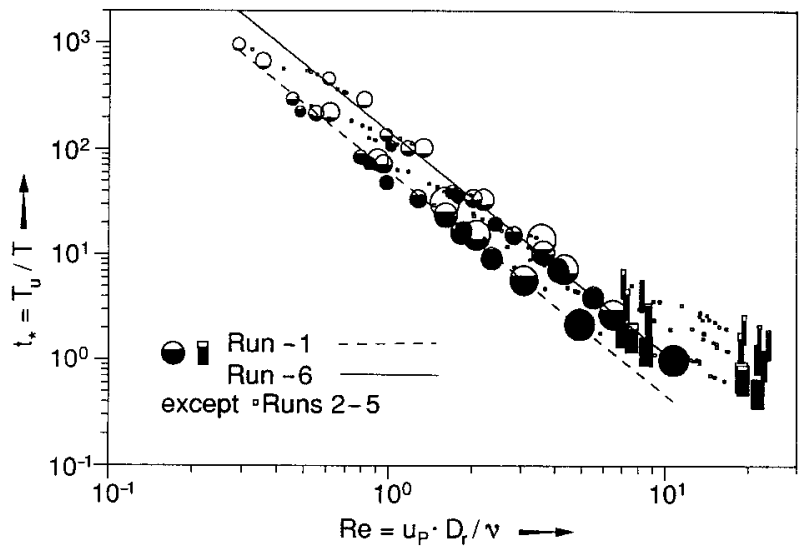

Fig. 6. A plot of dimensionless unit time $T_{u} / T$ versus particle Reynolds number $R e$ for zone I

Figure 5 is a plot of $\alpha$ against $D_{r} / D$. It is seen that the value of $\alpha$ for spheres diminishes with a reduction in the dimensionless draft while that for cylinders is constant.

The time required for a particle to migrate a unit distance at a velocity of $u_{p}$ is called unit time, $T_{u}$. The average $T_{u}$ in zone $\mathrm{I}$ is plotted against $R e$ in Fig. 6. Similar results are obtained for zones II and III (not shown). It is seen in the figure that like the drag coefficient $C_{d}, t_{*}$ decreases with a reduction in $R e$. For the same particle, $t_{*}$ varies with flow conditions (i.e. different runs). In other words, $t_{*}$ for a given particle diminishes with an increase in $R e$. The straight line in the figure is obtained by means of the least square mean method which is applied to twenty kids of spherical particles. The solid line (for $u_{p}=1.68 \mathrm{~cm} / \mathrm{s}$ ) and the broken line (for $u_{p}=0.80 \mathrm{~cm} / \mathrm{s}$ ) in Figs. 6 and 7 are obtained in a similar manner using the data of only spherical particles.

Both $C_{d}$ and $t_{*}$ diminish with an increase in $R e$, but at distinct decreasing gradient $t_{*}$ has a higher reduction rate than $C_{d}$. This implies that liquid friction on a particle is enhanced with an increase in the particle diameter and density ratio. Hence, it is appropriate to combine $C_{d}$ and $t_{*}$ into a single parameter which includes both the fluid flow condition, such as viscosity, and the particle condition, such as particle diameter and density ratio. Figure 7 illustrates a plot of $C_{d} / t_{*}$ versus $R e$. It is observed that $C_{d} / t_{*}$ increases with

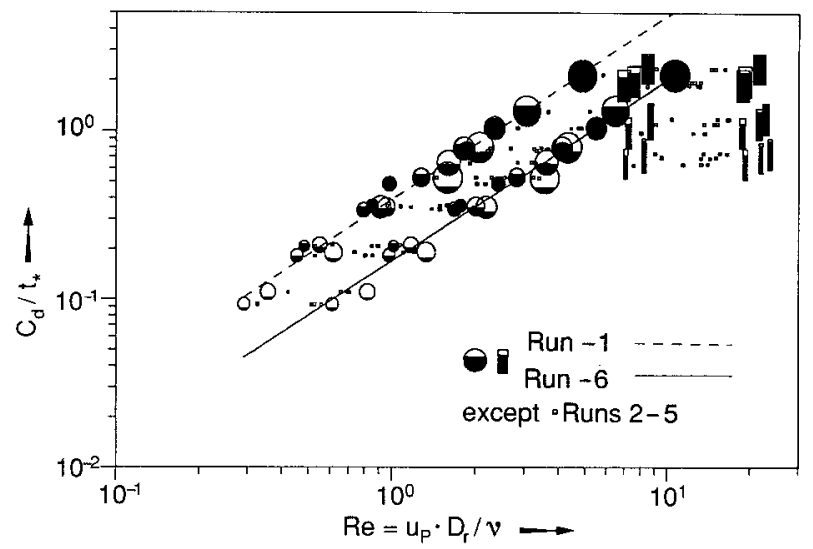

Fig. 7. Correlation of $C_{d} / t_{*}$ versus particle Reynolds number $R e$ for zone I

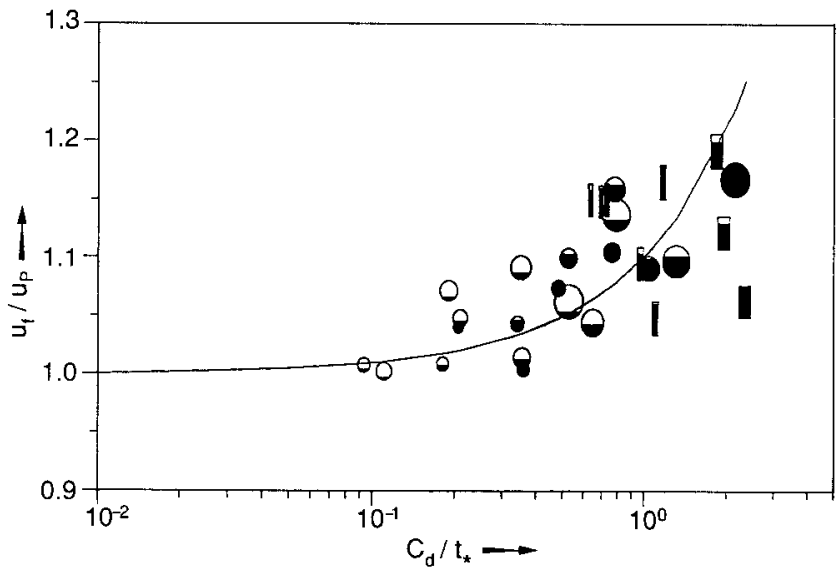

Fig. 8. Correlation of fluid-particle velocity ratio $u_{f} / u_{p}$ against $C_{d} / t_{*}$ in zone I

an increase in $R e$ for each run but does not change for each particle. Therefore, $C_{d} / t_{*}$ is an important nondimensional parameter which governs the dynamics of surface-floating particles.

The finite-difference Eqs. (14) and (15) were solved numerically for the fluid flow velocity $u_{f}$. The values of $u_{f, n+1}$ were calculated from Eqs. (14) and (15) using the measured values of $u_{p, n+1}$ and $u_{p, n}$. The one larger than $u_{p, n+1}$ was selected as the solution of $u_{f, n+1}$. Its average value in each zone was divided by average particle velocity to yield the velocity ration $u_{f} / u_{p}$ at that location. Figure 8 is a plot of $u_{f} / u_{p}$ versus $C_{d} / t_{*}$ in zone I for the average of all six runs. The solid line is the least square mean value. Similar plots for zones II and III are omitted in the interest of brevity. A correlation of $u_{f} / u_{p}$ against $R e$ (not shown) produces more data scattering than that of $u_{f} / u_{p}$ versus $C_{d} / t_{*}$. It is observed in Fig. 8 that $u_{f} / u_{p}$ increases as $R e$ is increased: $u_{f} / u_{p}=\exp \left(B C_{d} / t_{*}\right)$ where $B=0.0850$. The rate of enhancement in $u_{f} / u_{p}$ with $R e$ is more significant in run-6 for the fastest flow, than in run-1 for the slowest flow. This indicates a reduction in fluid flow would exert more effect on particle motion such as its direction and magnitude, acceleration, etc. 


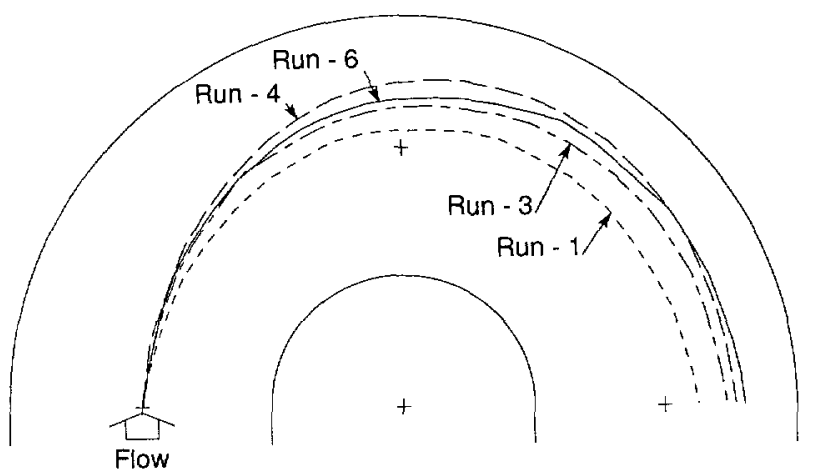

Fig. 9. Trajectory of particle No. 2

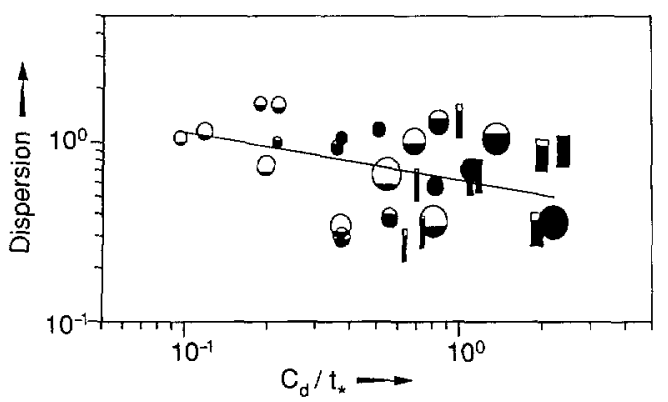

Fig. 10. Dispersion versus $C_{d} / t_{*}$ in the upstream half for run- 6

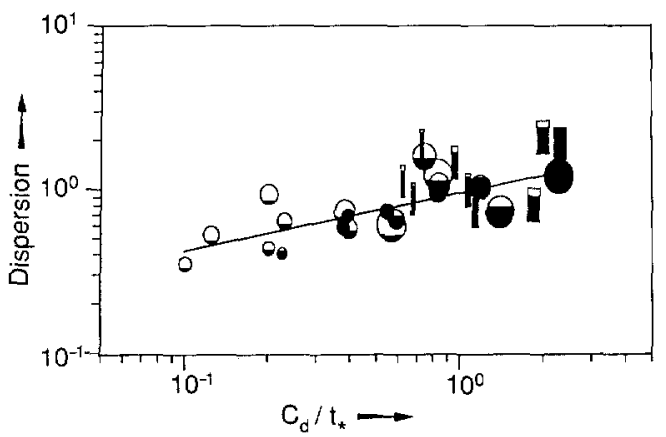

Fig. 11. Dispersion versus $C_{d} / t_{*}$ in the downstream half for run-6

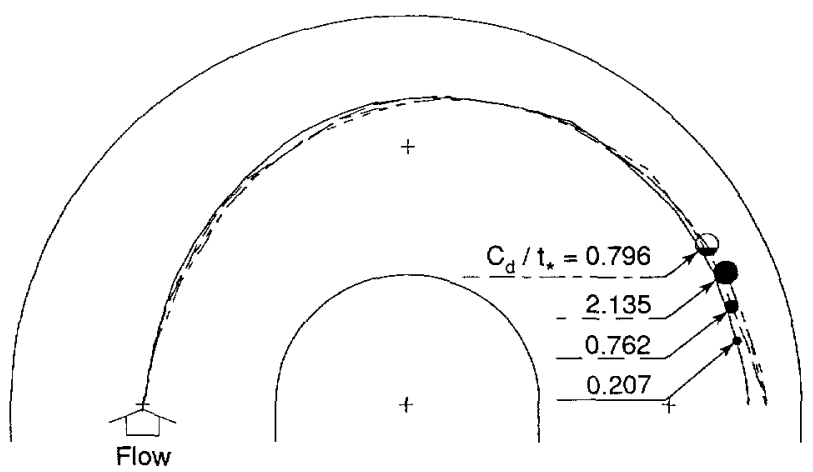

Fig. 12. Trajectory of various particles in the fastest flow case run- 6

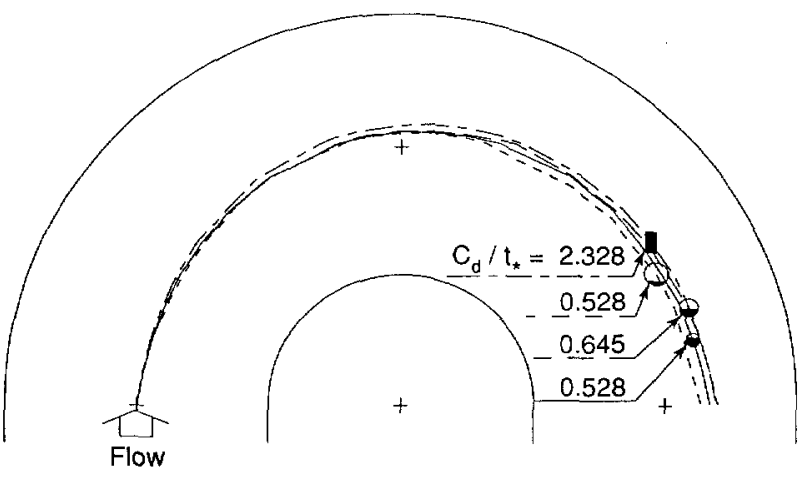

Fig. 13. Trajectory of various particles in the slowest flow case run-1

The particle trajectory changes with a change in the magnitude of $C_{d} / t_{*}$. Figure 9 depicts the trajectories of particle No. 2, a spherical particle with the smallest diameter. The trajectory of run-5 (not shown) practically coincides with those of runs 4 and 6 in the upstream and downstream halves, respectively. The trajectories of run-2 (not shown) and run-1 are almost the same in the entire semicircular channel. Run- 1 has the innermost trajectory which is nearly circular in shape. It shifts slightly; outward toward the end of the semicircular channel where the particle is accelerated. Of interest is the trajectory of run- 4 , which crosses that of run-6.

Next is to investigate how $C_{d} / t_{*}$ affects the particle trajectory. In each run, all trajectories of each particle under different density ratios and fluid flow conditions, $R_{n}$ 's are determined. Out of these $R_{n}$ 's the innermost trajectory $R_{\min }$ is selected as a reference, as shown in Fig. 3. A measure of derivation of $R_{n}$ from $R_{\min }$, called dispersion, is defined as $\sum_{n=1}^{N}\left(R_{n}-R_{\min }\right)^{2} / N$, where $N$ is the number of tests on each particle. Dispersion is plotted against $C_{d} / t_{*}$ for run-6 in Figs. 10 and 11 for the upstream and downstream halves, respectively. A smaller value of dispersion implies that the particle pathline is closer to the innermost trajectory. It is observed that for run- 6 dispersion decreases with an increase in $C_{d} / t_{*}$ in the upstream half, while the opposite is true in the downstream half. Similar trends appear in other runs. Figures 12 and 13 record the trajectories of various particles, characterized by different values of $C_{d} / t_{*}$ for the fastest and slowest flow cases, respectively. In general, dispersion of particle trajectories in the upstream half is small. At a higher velocity, dispersion remains small up to the vicinity of the channel exit, as seen in Fig. 12. However, Fig. 13 for slow flow indicates that larger particles with lower density ratios but same $C_{d} / t_{*}$ are more susceptible to flow conditions in the downstream half.

In summary, the particles with higher $C_{d} / t_{*}$ have a slightly inward trajectory in the upstream half compared to those of lower $C_{d} / t_{*}$. The action of centrifugal force causes particles to shift outward from a circular course. This phenomenon is observed in the particles of higher $C_{d} / t_{*}$, larger 
size or lower density ratio. However, a few exceptions are observed in the particles of same $C_{d} / t_{*}$ but lower density ratio, possibly due to the presence of a secondary stream induced by the effect of channel wall. For reference, the Dean's number in the fastest velocity case $(1.68 \mathrm{~cm} / \mathrm{s})$ is 209 , using the channel width as the characteristic length.

The study has revealed that the parameter $C_{d} / t_{*}$ is the base for comparing particle trajectories. If $C_{d} / t_{*}$ is larger, or the particle size is larger, or the density ratio is lower in the case of same $C_{d} / t_{*}$ particle trajectory would be more susceptible to the influence of various flow conditions. In other words, particles are less traceable. It should be noted that self rotation of particles during their migration with the liquid is not observed in the study.

\section{Conclusions}

An experimental study is performed on the dynamic characteristics of a single, surface-floating particle flowing in a planar, two-dimensional semicircular channel. A modified dynamics equation is derived for motion of surface-flotating particles. A new dimensionless parameter $C_{d} / t_{*}$ is disclosed which expresses both particle geometry and fluid flow conditions. It correlates the fluid-particle velocity ratio, particle trajectory and particle velocity. Results indicate that as the particle Reynolds number $R e$ increases, $C_{d} / t_{*}$ increases while both $C_{d}$ and $t_{*}$ diminish. The velocity ratio can be correlated by

$u_{f} / u_{p}=\exp \left(B C_{d} / t_{*}\right)$

where $B$ is an empirical constant.

\section{Acknowledgements}

The authors wish to express their gratitude to Professor M. Yano of Kobe University for his valuable suggestions during the initial stage of the study.

\section{References}

Brandone, B.; Bernard, P. 1971: Visualization par analogie hydraulique de l'ecoulement dans une grille d'aubes plane mobile. Rech. Aerospace No. 1971-2, 125-128

Clayton, B. R.; Massey, B. S. 1967: Flow visualization in water: a review of techniques. J. Sci. Instrum. 44, 2-11

Dimotakis, P. E.; Debussy, F. D.; Koochesfahani, M. M. 1981: Particle streak velocity field measurements in a two-dimensional mixing layer. Phys. Fluids 24, 995-999

Douglas, H. A.; Mason, P. J.; Hinch, E. J. 1972: Motion due to a moving internal heat source. J. Fluid Mech. 54, 469-480

Gharib, M.; Willert, C. 1989: Particle tracing: revised, advances in fluid mechanics measurements, lecture notes in engineering. (Ed. Gad-el-Hak, M.) Berlin Heidelberg New York: Springer, pp. $109-126$

Merzkirch, W. 1987: Flow visualization. New York: Academic Press, pp. $38-48$

Soo, S. L. 1967: Fluid dynamics of multiphase systems. Blaisdell Publishing, pp. $31-35$

Umeda, S.; Yano, M. 1988: Study of suspended particle motion in curved flow. 2nd International Symposium on FLUCOME, Sheffield, pp. $413-416$

Received February 6, 1991 